\title{
Evaluation of Cisplatin-Loaded Polymeric Micelles and Hybrid Nanoparticles Containing Poly(Ethylene Oxide)-Block- Poly(Methacrylic Acid) on Tumor Delivery
}

\author{
Andang Miatmoko1, Kumi Kawano1, Etsuo Yonemochi², Yoshiyuki Hattori ${ }^{*}$ \\ ${ }^{1}$ Department of Drug Delivery Research, Hoshi University, Shinagawa, Japan \\ ${ }^{2}$ Department of Physical Chemistry, Hoshi University, Shinagawa, Japan \\ Email: "yhattori@hoshi.ac.jp
}

Received 7 December 2015; accepted 10 January 2016; published 13 January 2016

Copyright (C) 2016 by authors and Scientific Research Publishing Inc.

This work is licensed under the Creative Commons Attribution International License (CC BY). http://creativecommons.org/licenses/by/4.0/

(c) (i) Open Access

\begin{abstract}
Particulate carriers such as polymeric micelles (PMs) and liposomes have been investigated to increase drug accumulation in tumors and reduce distribution to healthy tissues. In this study, we prepared PM and hybrid nanoparticles (HNPs) with poly(ethylene oxide)-block-poly(methacrylic acid) (PEO-b-PMAA) for loading cisplatin, and evaluated cisplatin release, cytotoxicity, and biodistribution in mice. PM composed of PEO-b-PMAA and HNPs composed of egg phosphatidylcholine (EPC)/PEO-b-PMAA at molar ratios of 50/2.8 (HNP-P5) and 50/50 (HNP-P50), respectively, were prepared by a nanoprecipitation method. The sizes of PM, HNP-P5, and HNP-P50 after inclusion of cisplatin were approximately 200,100 , and $55 \mathrm{~nm}$, respectively, and their entrapment efficiencies were approximately 44\% - 66\%. In the drug-release study, HNP-P5 and HNP-P50 showed reduced release of cisplatin compared with PM. Regarding the cytotoxic assay, HNP-P5 exhibited lower cytotoxicity for mouse Lewis lung carcinoma (LLC) and mouse colon carcinoma Colon 26 cells than PM and HNP-P50. In terms of biodistribution, PM could significantly improve blood circulation and tumor accumulation after intravenous injection into Colon 26 tumor-bearing mice compared with free cisplatin, but HNP-P5 and HNP-P50 did not. EPC in HNPs might be destabilized in the circulation, although it could reduce release of cisplatin in in vitro experiments. This study suggested that polymeric micelles composed of PEO-b-PMAA are a better carrier for cisplatin than hybrid nanoparticles composed of PEO-b-PMAA and EPC.
\end{abstract}

\section{Keywords}

Polymeric Micelles, Hybrid Nanoparticles, Cisplatin, PEO-b-PMAA

\footnotetext{
${ }^{*}$ Corresponding author.
}

How to cite this paper: Miatmoko, A., Kawano, K., Yonemochi, E. and Hattori, Y. (2016) Evaluation of Cisplatin-Loaded Polymeric Micelles and Hybrid Nanoparticles Containing Poly(Ethylene Oxide)-Block-Poly(Methacrylic Acid) on Tumor Delivery. Pharmacology \& Pharmacy, 7, 1-8. http://dx.doi.org/10.4236/pp.2016.71001 


\section{Introduction}

Cisplatin is a platinum (Pt) complex classified as an alkylating agent that has been widely used as monochemotherapy for cancers including lung, testicular, ovarian, bladder, head, neck, and esophageal cancer, or in combination with other drugs such as taxanes, gemcitabines, befuximab, bleomycin, etoposide, and vinca alkaloids [1]-[3]. Despite its clear benefits in clinical therapy, severe side-effects such as nephrotoxicity and distal neuropathy [4] [5], nausea, vomiting, anorexia, hearing loss [6] [7], and liver toxicities [8] have been reported. To overcome the problems of toxicities caused by cisplatin, particulate carriers such as polymeric micelles (PMs) and liposomes have been investigated to obtain high drug accumulation in tumors and reduce distribution to healthy tissues. Liposomal cisplatin, such as PEGylated liposomes e.g. Lipoplatin TM and SPI-077 [9] [10], or pH-sensitive PEGylated liposomes [11], could have high efficacy for the treatment of cancer and reduce the toxicity of cisplatin. However, the low solubility of cisplatin in water causes inefficient entrapment efficiency (7\% 19\%) of cisplatin into liposomes [12].

Cisplatin can interact with carboxylic acid in an aqueous environment [13]. Therefore, it can be loaded into PMs composed of a biodegradable block ionomers containing carboxylic acid such as poly(ethylene oxide)block-poly(methacrylic acid) (PEO-b-PMAA). The polymer has two different functional block segments, called a diblock polymer that can produce PM. Poly(ethylene oxide) (PEO) is a water soluble non-ionic polymer that is relatively non-toxic. It has the ability to reduce the recognition of PM by the immune system, resulting longer retention in plasma in the circulation [14]. In addition, poly(methacrylic acid) (PMAA) can form the inner core of PM by interaction with Pt [15]. Therefore, PM composed of PEO-b-PMAA might be a suitable carrier for cisplatin. However, an excess dilution upon systemic administration often causes the disintegration of PMs [16].

Hybrid nanoparticles (HNPs) consist of polymer and lipid components and have the properties of both lipid vesicles and polymeric micelles [17]. Thus, an additional lipid layer at the surface of hydrophobic block segment, such as with egg phosphatidylcholine (EPC) may be useful to protect the inner core and enhance particle stability. Therefore, in this study, to examine the possibility that incorporating cisplatin into HNPs could improve tumor accumulation of cisplatin via stabilization in the circulation, we prepared the PM composed of PEO-b-PMAA and HNPs composed of PEO-b-PMAA and EPC for cisplatin-loading, and compared cisplatin release, cytotoxicity, and biodistribution in mice.

\section{Materials and Methods}

\subsection{Materials}

Cisplatin was purchased from Wako Pure Chemical Industries Co., Ltd. (Tokyo, Japan). EPC (Coatsome NC-50) was obtained from NOF Corporation (Tokyo, Japan). PEO-b-PMAA (Mw of PEO = 7,500, Mn of PMAA = 11,000) was purchased from Polymer Source, Inc. (Quebec, Canada). All other reagents used in this study were the finest grade.

\subsection{Preparation of Polymeric Micelles and Hybrid Nanoparticles}

For preparation of PM, PEO-b-PMAA was dissolved in acetone. For preparation of HNP, the PEO-b-PMAA solution was added into EPC solution dissolved in methanol at a molar ratio of EPC/PEO-b-PMAA of 50/2.8 or 50/50 (HNP-P5 and HNP-P50, respectively). The solution of PEO-b-PMAA or mixture of EPC and PEO-bPMAA was added dropwise into water and stirred continuously for $30 \mathrm{~min}$ at room temperature. In order to remove the organic solvents, these mixtures were dialyzed against water using Spectra Por ${ }^{\circledR} 7$ dialysis membrane with molecular weight cut-off (MWCO) 2,000 (Spectrum Laboratories, Inc., CA, USA).

Cisplatin was incorporated into PM, HNP-P5 and HNP-P50 by direct mixing of cisplatin solution at pH 9 adjusted with ammonia solution at a molar ratio of carboxylic acid of PEO-b-PMAA/cisplatin of 1/2, and then incubated with shaking at $37^{\circ} \mathrm{C}$ for $48 \mathrm{~h}$. The concentration of carboxylic acid was determined by an acid-base titration method. Finally, free cisplatin was removed from the cisplatin-loaded PM, HNP-P5 and HNP-P50 by centrifugation at 2500 g for 20 min using an Amicon ${ }^{\circledR}$ Ultra filter (MWCO 30,000, Merck Millipore Ltd, Carrigtwohill, Ireland).

\subsection{Measurement of Particle Size, $\zeta$-Potential, and Entrapment Efficiency}

The particle sizes of PM, HNP-P5 and HNP-P50 were measured by the cumulant method using a light-scattering 
photometer (ELS-Z2, Otsuka Electronics Co, Ltd., Osaka, Japan) at $25^{\circ} \mathrm{C}$ after diluting the dispersion to an appropriate volume with water. The $\zeta$-potentials were measured by electrophoresis light-scattering methods using ELS-Z2 at $25^{\circ} \mathrm{C}$ after diluting the dispersion to an appropriate volume with water. For calculating the entrapment efficiency of cisplatin, cisplatin was extracted from PM, HNP-P5, and HNP-P50 by solubilizing lipid layer using the Bligh and Dyer method [18]. Briefly, $20 \mu \mathrm{L}$ of PM, HNP-P5, or HNP-P50 solution was mixed with $180 \mu \mathrm{L} 0.1 \mathrm{~N} \mathrm{HCl}$ solution. A $750 \mu \mathrm{L}$ aliquot of a solution of methanol: chloroform (1:2), $250 \mu \mathrm{L}$ chloroform and $250 \mu \mathrm{L} 0.1 \mathrm{~N} \mathrm{HCl}$ was added and then centrifuged to separate the aqueous (upper) and organic solvent (lower) phases. The cisplatin content of the upper phase was measured with a high-performance liquid chromatography (HPLC) system (Shimadzu Co. Kyoto, Japan) composed of an LC-10 ATVP pump, an SIL-10 AF autoinjector, and an SPD-10 AVP UV detector at an absorbance at $306 \mathrm{~nm}$. The upper phase was applied to an anion exchange column Inertsil $\mathrm{AX}^{\circledR}(250 \mathrm{~mm} \times 4.6 \mathrm{~mm}$, GL Sciences, Tokyo, Japan) equilibrated in a mixture of 8:40:10:20 (v/v) of ethyl acetate, methanol, water and $N, N$-dimethylformamide at a flow rate of $1 \mathrm{~mL} / \mathrm{min}$. The entrapment efficiency (EE\%) of cisplatin in PM, HNP-P5 and HNP-P50 was calculated using the following equation.

$\mathrm{EE} \%=($ Amount of drug entrapped $) /($ Amount of drug added $) \times 100$.

\subsection{Drug Release Study}

The release studies of cisplatin were performed by placing $200 \mu \mathrm{L}$ of PM, HNP-P5, or HNP-P50 solution into Spectra $\operatorname{Por}^{\circledR} 7$ dialysis tubing with MWCO 3500 (Spectrum Laboratories, Inc.). The PM and HNPs were then immersed in $50 \mathrm{~mL}$ of phosphate-buffered saline (PBS; pH 5.5 or 7.4) with continuous stirring in a water bath at $37^{\circ} \mathrm{C}$. At various time points, $200 \mu \mathrm{L}$ aliquots were withdrawn from the outer aqueous solution and replaced with $200 \mu \mathrm{L}$ of PBS. The Pt concentration was measured by a graphite furnace atomic absorption spectrophotometry (GF-AAS) method after diluting with $0.1 \mathrm{~N} \mathrm{HCl}$ to an appropriate volume. The analysis program of the GF-AAS involved three steps: 1) a drying stage at $80^{\circ} \mathrm{C}-100^{\circ} \mathrm{C}$ for $40 \mathrm{~s}, 2$ ) an ashing stage at $800^{\circ} \mathrm{C}$ for $30 \mathrm{~s}, 3$ ) an atomization stage by heating at $3000^{\circ} \mathrm{C}$ for $7 \mathrm{~s}$, and then cooling down. The concentration of Pt was measured at $265.9 \mathrm{~nm}$ with a slit bandwidth of $0.4 \mathrm{~nm}$, and then converted into cisplatin concentration. Wurster correction was used for calculating the cumulative amount of cisplatin released.

\subsection{In Vitro Cytotoxic Assay}

Murine Lewis lung carcinoma (LLC) and colon carcinoma Colon 26 cells were obtained from the Cell Resource Center for Biomedical Research, Tohoku University (Miyagi, Japan). The cells were cultured in RPMI-1640 medium with $10 \%$ heat-inactivated fetal bovine serum and kanamycin $(100 \mu \mathrm{g} / \mathrm{mL})$ in a humidified atmosphere containing $5 \% \mathrm{CO}_{2}$ at $37^{\circ} \mathrm{C}$. For the in vitro cytotoxic assay, LLC and Colon 26 cells were seeded separately at a density of $1 \times 10^{4}$ cells per well in 96-well plates, respectively, and maintained in the medium for $24 \mathrm{~h}$ before treatment.

To examine the cytotoxicity of cisplatin, the cells were treated with medium containing various concentrations of cisplatin in PM, HNP-P5, and HNP-P50, and they were then incubated for $48 \mathrm{~h}$. After treatment, the cell number was determined using a Cell Counting Kit-8 (Dojindo Laboratories, Kumamoto, Japan). Cell viability (\%) was expressed relative to the absorbance of untreated cells at $450 \mathrm{~nm}$.

\subsection{In Vivo Biodistribution Study}

All animal experiments were performed with approval from the Institutional Animal Care and Use Committee of Hoshi University. To generate Colon 26 tumors, $1 \times 10^{6}$ cells were inoculated subcutaneously into the flank of female BALB/c mice (female, 6 weeks old, Sankyo Lab. Service Corp., Tokyo, Japan). After the tumor size had reached $100 \mathrm{~mm}^{3}$, PM, HNP-P5, and HNP-P50 were administered intravenously via the tail vein at a dose equivalent to $4 \mathrm{mg}$ cisplatin per $\mathrm{kg}$. Twenty-four hours after injection, blood was collected in heparinized tubes by decapitation to obtain plasma, and the tumor and kidneys were excised.

For analysis of Pt levels, plasma, tumor, and kidneys were digested with concentrated $\mathrm{HNO}_{3}$, and then heated at $70^{\circ} \mathrm{C}$ for $1 \mathrm{~h}$ followed by heating at $120^{\circ} \mathrm{C}$ overnight. The concentration of Pt was measured by GF-AAS method after diluting with $0.1 \mathrm{~N} \mathrm{HCl}$ in appropriate volume as described above section, and calculated as $\mu \mathrm{g} \cdot \mathrm{Pt} / \mathrm{mL}$ plasma or $\mu \mathrm{g} \cdot \mathrm{Pt} / \mathrm{g}$ tissue. 


\subsection{Statistical Analysis}

All data were produced in replicates and presented as the mean $\pm \mathrm{SD}$. To evaluate the significance of differences, data were analyzed by one-way ANOVA, followed by Tukey’s HSD post-hoc test, with $P$ values less than 0.05 considered as statistically significant.

\section{Results and Discussion}

\subsection{Characterizations of Hybrid Nanoparticles}

PM, HNP-P5, and HNP-P50 were prepared by the nanoprecipitation method. PM consisted of 100\% PEO-bPMAA, and HNP-P5 and HNP-P50 contained at 5.3 and 50 mol\% PEO-b-PMAA with EPC, respectively. EPC comprises a polar head of choline, with fatty acid forming the double tail chains [19]. These lipophilic chains are expected to associate with the hydrophobic segment of PEO-b-PMAA to form an additional protective layer of the micelle and avoid micelle breakage during the excessive dilution in body fluids.

The sizes of PM, HNP-P5, and HNP-P50 before inclusion of cisplatin were approximately $220 \mathrm{~nm}, 110 \mathrm{~nm}$, and $65 \mathrm{~nm}$, respectively, and their $\zeta$-potentials were approximately $-21 \mathrm{mV},-13 \mathrm{mV}$, and $-17 \mathrm{mV}$, respectively (Table 1). Generally, the size of PM was smaller than the vesicles of liposomes [20]. However, the size of PM was large compared with HNP-P5 and HNP-P50. It has been reported previously that divalent cation metal is needed to form self-assembly ionomer complexes with PEO-b-PMAA [21]. The large size of PM may relate to the hydration of PEO-b-PMAA by water without divalent cation metals. In contrast, in HNPs, the hydrophobicity of EPC might change the amphiphatic balance of PEO-b-PMAA.

After cisplatin loading, the particle sizes of PM, HNP-P5, and HNP-P50 were not significantly changed, but their $\zeta$-potentials were increased (Table 1 ), indicating that the negatively charged carboxylic acid of PMAA interacted electrostatically with cisplatin. In contrast, increasing the EPC content in HNP slightly reduced entrapment efficiency (Table 1), suggesting that the high content of PEO-b-PMAA in PM and HNP-P50 could interact well with cisplatin.

\subsection{In Vitro Drug-Release Study of Hybrid Nanoparticles}

The in vitro profiles of cisplatin release from PM, HNP-P5, and HNP-P50 were evaluated by immersing nanoparticles in PBS (pH 5.5 and 7.4). Decreasing the $\mathrm{pH}$ from 7.4 to 5.5 increased the release of cisplatin from PM, HNP-P5, and HNP-P50 (Figure 1(a), Figure 1(b)). It has been reported that dissociation of cisplatin from PMAA depended on the $\mathrm{pH}$ and ionic strength of the environment [15]. HNP-P5 and HNP-P50 showed low release of cisplatin at approximately $40 \%$ and $67 \%$, respectively, of the cumulative dose over $120 \mathrm{~h}$ in PBS pH 5.5, whereas PM exhibited the highest release of up to 90\% (Figure 1(b)). HNP-P5 exhibited reduced cisplatin release compared with HNP-P50, indicated that increasing EPC content in HNPs reduced cisplatin release. The presence of the lipid layer could limit water diffusion into the inner part of particles [22]; therefore, the barrier of EPC in HNPs might reduce cisplatin release.

\subsection{In Vitro Cytotoxicity Assay}

Next, we evaluated the cytotoxicity of PM, HNP-P5, and HNP-P50 on Colon 26 and LLC cells by 48 h exposure. PM and HNP-P50 had similar cytotoxic effects on Colon 26 ( $\mathrm{IC}_{50}=1.79$ and $1.71 \mu \mathrm{g} / \mathrm{mL}$, respectively) (Figure 2(a)) and LLC cells (IC50 $=0.42$ and $0.87 \mu \mathrm{g} / \mathrm{mL}$, respectively) (Figure 2(b)) compared with cisplatin solution

Table 1. Characterization of polymeric micelle (PM) and hybrid nanoparticles (HNPs) before and after inclusion of cisplatin.

\begin{tabular}{cccccc} 
Formulation & \multicolumn{2}{c}{ Particle size $(\mathrm{nm})^{\mathrm{a})}$} & \multicolumn{2}{c}{$\zeta$-potential $(\mathrm{mV})^{\mathrm{a})}$} & $\begin{array}{c}\text { Entrapment } \\
\text { efficiency }(\%)^{\mathrm{a}}\end{array}$ \\
\cline { 2 - 5 } & Before & After & Before & After & $66.1 \pm 4.0$ \\
PM & $221.7 \pm 186.4$ & $203.7 \pm 194.3$ & $-21.3 \pm 4.5$ & $0.5 \pm 1.7$ & $44.5 \pm 2.4$ \\
HNP-P5 & $107.7 \pm 9.4$ & $103.3 \pm 7.7$ & $-13.4 \pm 0.7$ & $2.2 \pm 3.4$ & $56.2 \pm 14.3$ \\
HNP-P50 & $65.4 \pm 5.5$ & $54.6 \pm 4.7$ & $-16.9 \pm 1.3$ & $2.5 \pm 2.3$ & \\
\hline
\end{tabular}

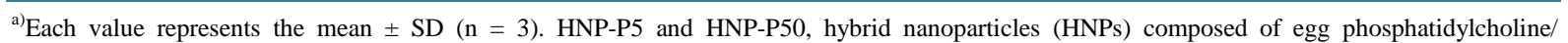
poly(ethylene oxide)-block-poly(methacrylic acid) at molar ratios of 50/2.8 and 50/50, respectively. 
(a)

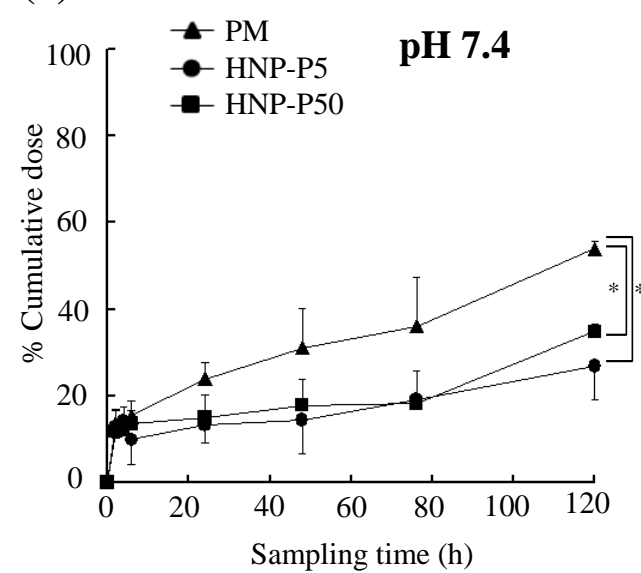

(b)

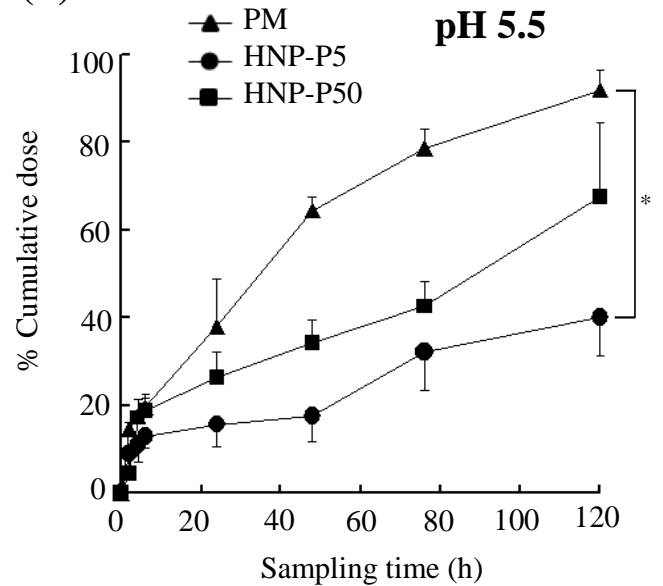

Figure 1. Profiles of cisplatin release from polymeric micelle (PM) and hybrid nanoparticles (HNPs) composed of egg phos phatidylcholine/poly(ethylene oxide)-block-poly(methacrylic acid) at molar ratios of 50/2.8 (HNP-P5) and 50/50 (HNP-P50) in phosphate-buffered saline (PBS), $\mathrm{pH} 7.4$ (a) and $\mathrm{pH} 5.5$ (b) at $37^{\circ} \mathrm{C}$. Each value represents mean $\pm \mathrm{SD}(\mathrm{n}=3) .{ }^{*} P<0.05$.

\section{(a)}

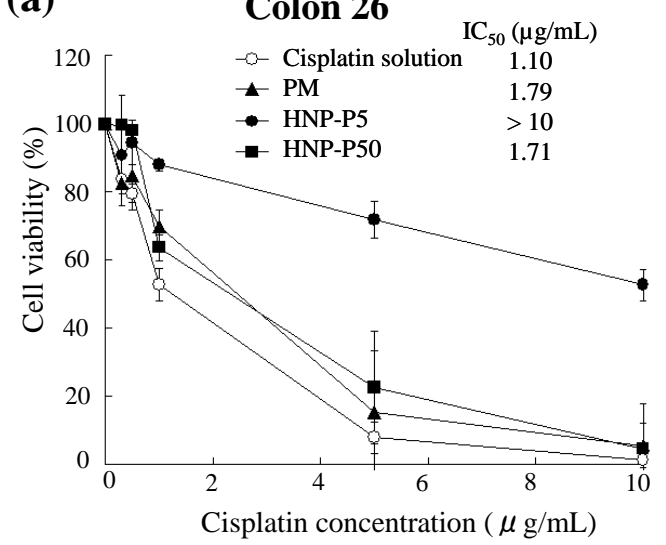

(b)

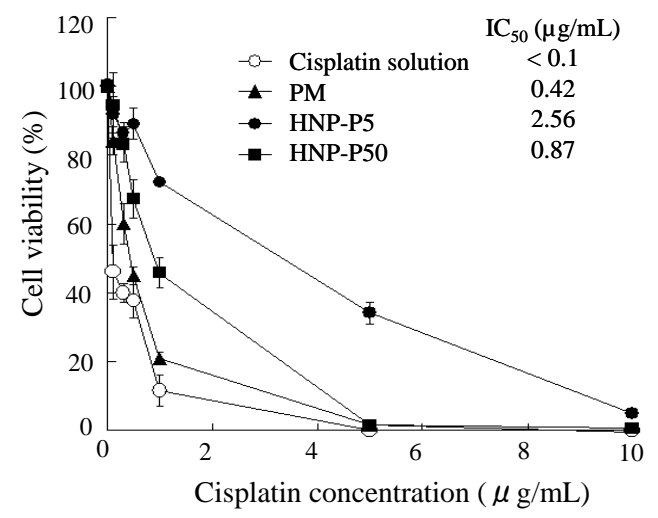

(c)

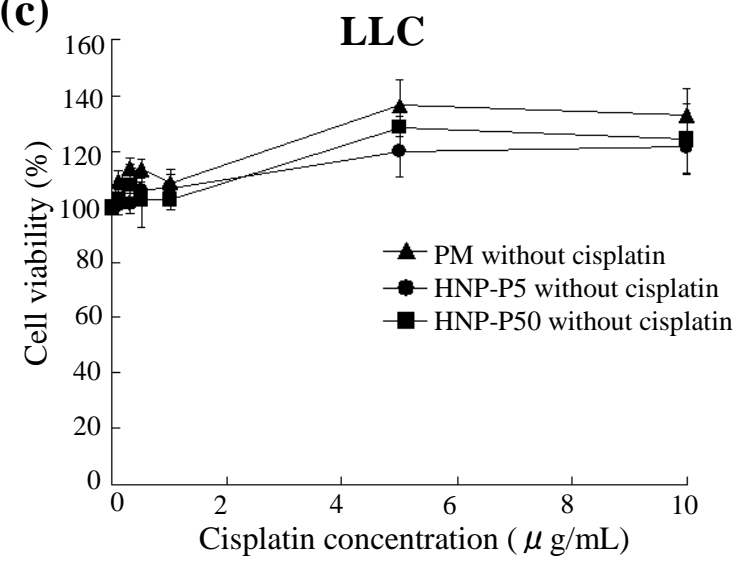

Figure 2. In vitro cytotoxicity of polymeric micelle (PM) and hybrid nanoparticles (HNPs) composed of egg phosphatidylcholine/poly(ethylene oxide)-block-poly(methacrylic acid) at molar ratios of 50/2.8 (HNP-P5) and 50/50 (HNP-P50) with Colon 26 cells (a) and Lewis lung carcinoma (LLC) cells (b), (c)). Cisplatin-loaded PM, HNP-P5, and HNP-P50 were incubated at various concentrations of cisplatin ((a) and (b)) for $48 \mathrm{~h}$. In (c), PM, HNP-P5, and HNP-P50 without cisplatin were incubated with LLC cells. The concentration of cisplatin indicates the same amount of PM or HNPs with cisplatin-loaded $\mathrm{PM}$ or HNPs. Each value represents mean $\pm \mathrm{SD}(\mathrm{n}=3)$. 
( IC $_{50}=1.10 \mu \mathrm{g} / \mathrm{mL}$ for Colon 26 and $<0.1 \mu \mathrm{g} / \mathrm{mL}$ for LLC cells). In contrast, HNP-P5 decreased cytotoxic effects ( $\mathrm{IC}_{50} \geq 10 \mu \mathrm{g} / \mathrm{mL}$ for Colon 26 and $2.56 \mu \mathrm{g} / \mathrm{mL}$ for LLC) compared with PM and HNP-P50. These results correlated well with the slow release of cisplatin from HNPs. Furthermore, to confirm the effect of carriers on the cell viability, LLC cells were exposed to PM, HNP-P5, and HNP-P50 without cisplatin, and no effects were observed on the cell viability (Figure 2(c)).

\subsection{In Vivo Biodistribution after Intravenous Injection}

Finally, we evaluated the biodistribution $24 \mathrm{~h}$ after injection of cisplatin-loaded PM, HNP-P5, and HNP-P50 on Colon 26 tumor-bearing mice. Compared with cisplatin solution ( $0.1 \mu \mathrm{g} \cdot \mathrm{Pt} / \mathrm{mL}$ plasma), which was almost cleared from the systemic circulation, the concentration of Pt in plasma after injection of PM remained at 5.3 $\mu \mathrm{g} \cdot \mathrm{Pt} / \mathrm{mL}$ at $24 \mathrm{~h}$ (Figure 3(a)). Higher tumor accumulation of cisplatin was also observed with PM (1.7 $\mu \mathrm{g} \cdot \mathrm{Pt} / \mathrm{g}$ tumor) than HNP-P5 or HNP-P50 (0.5 and $0.9 \mu \mathrm{g} \cdot \mathrm{Pt} / \mathrm{g}$ tumor, respectively) (Figure 3(b)). This suggested that PM could significantly improve tumor accumulation of cisplatin by prolonged circulation in the bloodstream (EPR effect), compared with cisplatin solution. Although HNP-P5 and HNP-P5 did not significantly increase cisplatin levels in plasma and tumors compared with cisplatin solution, they were still better than cisplatin solution. We speculated that EPC in HNPs might be destabilized in the circulation by interaction with blood components, although it could reduce the release of cisplatin in the in vitro experiments (Figure 1 and Figure 2). In contrast, the accumulation of cisplatin in the kidneys after injection of PM, HNP-P5, and HNP-P50 was low and did not show significant differences (Figure 3(c)). We also prepared other types of HNPs composed of EPC/ PEO-b-PMAA/sodium deoxycholate or EPC/PEO-b-PMAA/Tween 80 at a molar ratio of 50/2.8/5, and evaluated their biodistribution at $24 \mathrm{~h}$ after intravenous injection into mice; however, their cisplatin levels in plasma (1.1 and $0.8 \mu \mathrm{g} \cdot \mathrm{Pt} / \mathrm{mL}$, respectively) and tumor ( 0.3 and $0.3 \mu \mathrm{g} \cdot \mathrm{Pt} / \mathrm{g}$ tumor, respectively) were low compared with PM (data not shown). In HNPs, further improvement might be required to obtain better stability in vivo. Using lipids with higher phase transition temperature such as HSPC for the formulation of HNPs may be able to improve in vivo tumor delivery.

\section{Conclusion}

In the present study, we found that hybrid nanoparticles composed of PEO-b-PMAA and EPC showed improved in vitro stability; however, they did not increase circulatory concentration or tumor accumulation of cisplatin after intravenous injection compared with PM composed of PEO-b-PMAA. The present work suggested that further improvement of the formulation of hybrid nanoparticles might be required to obtain better stability in vivo.

(a)

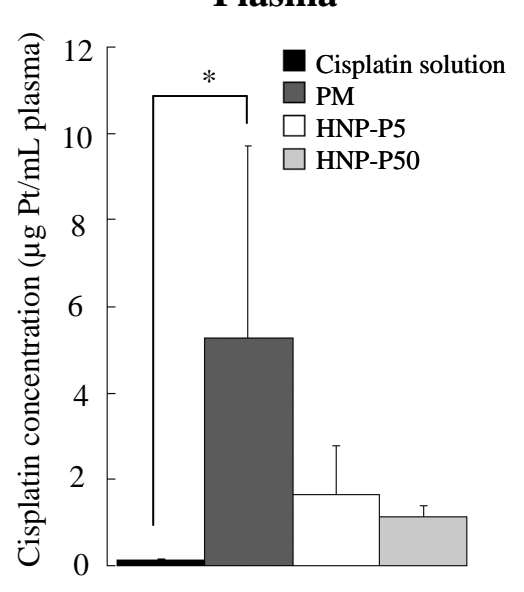

(b)

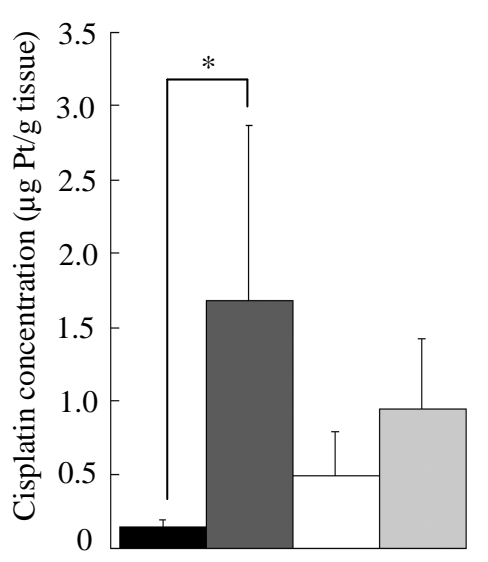

(c)

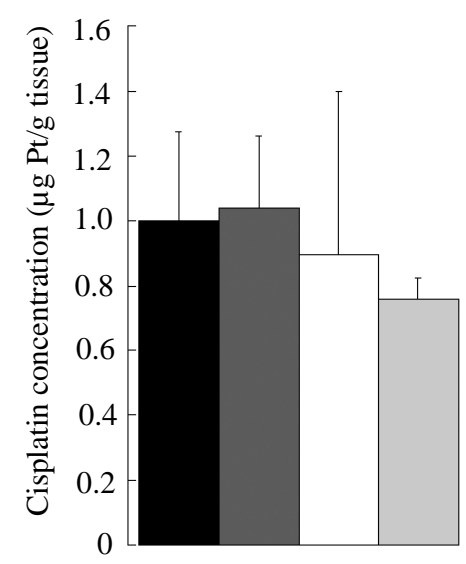

Figure 3. Biodistribution of cisplatin in Colon 26 tumor-bearing mice at $24 \mathrm{~h}$ after a single intravenous injection of polymeric micelle (PM) or hybrid nanoparticles (HNPs) composed of egg phosphatidylcholine/poly(ethylene oxide)-block-poly(methacrylic acid) at molar ratios of 50/2.8 (HNP-P5) and 50/50 (HNP-P50) at doses equal to 4 mg cisplatin per kg. Each value represents mean $\pm \mathrm{SD}(\mathrm{n}=4){ }^{*} \mathrm{P}<0.05$. 


\section{Conflicts of Interest}

The authors declare that they have no potential conflict of interests by any means to any institution, organization while publishing this study.

\section{References}

[1] Kelland, L. (2007) The Resurgence of Platinum-Based Cancer Chemotherapy. Nature Reviews Cancer, 7, $573-584$. http://dx.doi.org/10.1038/nrc2167

[2] Homesley, H.D., Bundy, B.N., Hurteau, J.A. and Roth, L.M. (1999) Bleomycin, Etoposide, and Cisplatin Combination Therapy of Ovarian Granulosa Cell Tumors and Other Stromal Malignancies: A Gynecologic Oncology Group Study. Gynecologic Oncology, 72, 131-137. http://dx.doi.org/10.1006/gyno.1998.5304

[3] Ray-Coquard, I., Biron, P., Bachelot, T., Guastalla, J.P., Catimel, G., Merrouche, Y., Droz, J.P., Chauvin, F. and Blay, J.Y. (1998) Vinorelbine and Cisplatin (CIVIC Regimen) for the Treatment of Metastatic Breast Carcinoma after Failure of Anthracycline- and/or Paclitaxel-Containing Regimens. Cancer, 82, 134-140. http://dx.doi.org/10.1002/(SICI)1097-0142(19980101)82:1<134::AID-CNCR16>3.0.CO;2-3

[4] Miller, R.P., Tadagavadi, R.K., Ramesh, G. and Reeves, W.B. (2010) Mechanisms of Cisplatin Nephrotoxicity. Toxins (Basel), 2, 2490-2518. http://dx.doi.org/10.3390/toxins2112490

[5] Quasthoff, S. and Hartung, H.P. (2002) Chemotherapy-Induced Peripheral Neuropathy. Journal of Neurology, 249, 917. http://dx.doi.org/10.1007/PL00007853

[6] Kurihara, N., Kubota, T., Hoshiya, Y., Otani, Y., Ando, N., Kumai, K. and Kitajima, M. (1996) Pharmacokinetics of Cis-Diamminedichloroplatinum (II) Given as Low-Dose and High-Dose Infusions. Journal of Surgical Oncology, 62, 135-138. http://dx.doi.org/10.1002/(SICI)1096-9098(199606)62:2<135::AID-JSO10>3.0.CO;2-7

[7] Simon, T., Hero, B., Dupuis, W., Selle, B. and Berthold, F. (2002) The Incidence of Hearing Impairment after Successful Treatment of Neuroblastoma. Klinische Padiatrie, 214, 149-152. http://dx.doi.org/10.1055/s-2002-33179

[8] El-Sayyad, H., Ismail, M.F., Shalaby, F.M., Abou-El-Magd, R.F., Gaur, R.L., Fernando, A., Raj, M.H. and Ouhtit, A. (2009) Histopathological Effects of Cisplatin, Doxorubicin and 5-Flurouracil (5-FU) on the Liver of Male Albino Rats. International Journal of Biological Sciences, 5, 466-473. http://dx.doi.org/10.7150/ijbs.5.466

[9] Newman, M.S., Colbern, G.T., Working, P.K., Engbers, C. and Amantea, M.A. (1999) Comparative Pharmacokinetics, Tissue Distribution, and Therapeutic Effectiveness of Cisplatin Encapsulated in Long-Circulating, Pegylated Liposomes (SPI-077) in Tumor-Bearing Mice. Cancer Chemotherapy and Pharmacology, 43, 1-7. http://dx.doi.org/10.1007/s002800050855

[10] Slingerland, M., Guchelaar, H.J. and Gelderblom, H. (2012) Liposomal Drug Formulations in Cancer Therapy: 15 Years along the Road. Drug Discovery Today, 17, 160-166. http://dx.doi.org/10.1016/j.drudis.2011.09.015

[11] Leite, E.A., Giuberti Cdos, S., Wainstein, A.J., Wainstein, A.P., Coelho, L.G., Lana, A.M., Savassi-Rocha, P.R. and De Oliveira, M.C. (2009) Acute Toxicity of Long-Circulating and pH-Sensitive Liposomes Containing Cisplatin in Mice after Intraperitoneal Administration. Life Sciences, 84, 641-649. http://dx.doi.org/10.1016/j.lfs.2009.02.002

[12] Carvalho Jr., A.D., Vieira, F.P., Melo, V.J., Lopes, M.T., Silveira, J.N., Ramaldes, G.A., Garnier-Suillerot, A., Pereira-Maia, E.C. and Oliveira, M.C. (2007) Preparation and Cytotoxicity of Cisplatin-Containing Liposomes. Brazilian Journal of Medical and Biological Research, 40, 1149-1157. http://dx.doi.org/10.1590/S0100-879X2006005000125

[13] Cepeda, V., Fuertes, M.A., Castilla, J., Alonso, C., Quevedo, C. and Perez, J.M. (2007) Biochemical Mechanisms of Cisplatin Cytotoxicity. Anti-Cancer Agents in Medicinal Chemistry, 7, 3-18. http://dx.doi.org/10.2174/187152007779314044

[14] Kwon, G.G. and Katoka, K. (2012) Block Copolymer Micelles as Long-Circulating Vehicles. Advanced Drug Delivery Reviews, 64, 237-245. http://dx.doi.org/10.1016/j.addr.2012.09.016

[15] Mori, H. and Muller, A.H.E. (2003) New Polymeric Architectures with (Meth)acrylic Acid Segments. Progress in Polymer Science, 28, 1403-1439. http://dx.doi.org/10.1016/S0079-6700(03)00076-5

[16] Owen, S.C., Chan, D.P.Y. and Shoichet, M.S. (2012) Polymeric Micelle Stability. Nano Today, 7, 53-65. http://dx.doi.org/10.1016/j.nantod.2012.01.002

[17] Mandal, B., Bhattacharjee, H., Mittal, N., Sah, H., Balabathula, P., Thoma, L.A. and Wood, G.C. (2013) Core-ShellType Lipid-Polymer Hybrid Nanoparticles as a Drug Delivery Platform. Nanomedicine, 9, 474-491. http://dx.doi.org/10.1016/j.nano.2012.11.010

[18] Bligh, E.G. and Dyer, W.J. (1959) A Rapid Method of Total Lipid Extraction and Purification. Canadian Journal of Biochemistry and Physiology, 37, 911-917. http://dx.doi.org/10.1139/059-099

[19] Walker, R.A., Gruetzmacher, J.A. and Richmond, G.L. (1998) Phosphatidylcholine Monolayer Structure at a Liq- 
uid-Liquid Interface. Journal of the American Chemical Society, 120, 6991-7003. http://dx.doi.org/10.1021/ja980736k

[20] Nishiyama, N. and Kataoka, K. (2006) Current State, Achievements, and Future Prospects of Polymeric Micelles as Nanocarriers for Drug and Gene Delivery. Pharmacology \& Therapeutics, 112, 630-648. http://dx.doi.org/10.1016/j.pharmthera.2006.05.006

[21] Kim, J.O., Nukolova, N.V., Oberoi, H.S., Kabanov, A.V. and Bronich, T.K. (2009) Block Ionomer Complex Micelles with Cross-Linked Cores for Drug Delivery. Polymer Science Series A: Polymer Physics, 51, 708-718. http://dx.doi.org/10.1134/S0965545X09060169

[22] Hadinoto, K., Sundaresan, A. and Cheow, W.S. (2013) Lipid-Polymer Hybrid Nanoparticles as a New Generation Therapeutic Delivery Platform: A Review. European Journal of Pharmaceutics and Biopharmaceutics, 85, 427-443. http://dx.doi.org/10.1016/j.ejpb.2013.07.002 